\title{
EFFICIENCY OF METHYL EUGENOL DILUTED WITH CERTAIN OILS ON FIELD ATTRACTION OF THE PEACH FRUIT FLY, BACTROCERA ZONATA
}

\author{
MOSTAFA M. EL-METWALLY and ALI A. AMIN
}

Plant Protection Research Institute, ARC, Dokki, Giza

(Manuscript received 20 April 2015)

\begin{abstract}
Abstrac
$\mathrm{T}$ he peach fruit fly, Bactrocera zonata (Saunders) is considered as one of the most significant insects that attack commercial fruits in Egypt. The fibered blocks treated with methyl eugenol and Sumithion $(4: 1)$ is one of the most applicable methods that used through Male Annihilation Technique to control this insect. This study was carried out in order to minimize the economic cost by decreasing the consumed quantities of methyl eugenol in control procedures application through two successive trails. The $1^{\text {st }}$ trail aimed to evaluate different doses of methyl eugenol in comparison with the standard dose in fiber blocks. The mean no. of attracted males of tested treatments $(8,6,4$ and 2 $\mathrm{cm}^{3}$ ) varied significantly and averaged $13.46,18.55,8.46$ and 4.99 flies / week, respectively, at Fayom governorate. At Dakhlia governorate, the mean no. of attracted males of the tested treatments were 55.67, 34.60, 25.20 and 13.51 flies / week, respectively. The $2^{\text {nd }}$ trail was contributed to evaluate the field efficiency of methyl eugenol diluted by certain oils on attraction of B. zonata males. The tested treatments were; (A) Methyl eugenol $98 \%$, (B) Methyl eugenol $75 \%+$ corn oil 25\%, (C)Methyl eugenol $50 \%+$ corn oil 50\%, (D) Methyl eugenol $75 \%+$ sunflower oil $25 \%$, (E) Methyl eugenol $50 \%+$ sunflower oil 50\%, (F) Methyl eugenol $75 \%$ + paraffin oil $25 \%$, and (G) Methyl eugenol $50 \%+$ paraffin oil $50 \%$. At Fayom governorate, the treatments $\mathrm{G}, \mathrm{F}$ and $\mathrm{B}$ were highly significant in capturing averaging $35.85,24.86$ and 24.78 flies / week, respectively. At Menofia governorate, the general means were highly insignificant varied whereas the treatments $A, G$ and $C$ were the highest insignificantly attracting with the respective means $17.83,16.58$ and 12.55 flies/ week. The obtained results in both governorates revealed the possibility of paraffin oil utilization as diluent for methyl eugenol on fiber block treatments without negative effect on attraction of $B$. zonata, and hence minimizing the economic costs of peach fruit fly control applications.
\end{abstract}

\section{INTRODUCTION}

The peach fruit fly, Bactrocera zonata (Saunders) is one of the most harmful tephritid insects that infesting many commercial fruit hosts. Methyl eugenol (4-allyl1,2-dimethoxybenzene) is a kairomone (Metcalf and Metcalf, 1992) that is attractive to the males of $B$. zonata and has been tested to suppress the fly population through male annihilation technique (MAT) concept of insect control (Qureshi et al., 1981). 
Male annihilation technique (MAT) is a fruit fly control method removes males of insect, thus reducing male population. This disturbs male: female ratio and reducing the insect's chances of mating and females produce very few progeny. As a result, the wild population in the target area declines and the insects are eradicated at the end (Zaheeruddin, 2007).

Soaked-wooden killer blocks are considered one of the most preferable methods of MAT for fruit fly Bactrocera species(Afzal \& Javed 2001 \& Afzal et al., 2001). Fibered blocks were treated by methyl eugenol and Suumithion as a toxicant were the highest efficient for attracting and killing the highest numbers of $B$. zonata males for up to 4 weeks (Ghanim et al., 2010 and Amin, 2013). The ovipositional behavior of the gravid Queensland fruit fly, Dacus tryoni (Froggat) was studied by diluting odour of vapours certain pure chemicals typical to those emitting of ripe fruits with paraffin oil (Eisemann and Rice 1992).

Dilution of methyl eugenol in paraffin oil till $50 \%$ in MAT by using spinosad (as an insecticide) had a high effect against B. zonata population (Ghanim, 2013). Dilutions of methyl eugenol with paraffin oil may cause regular release of volatiles in its mixture and hence maintains potency for a longer time, methyl eugenol diluted with paraffin oil up to $75 \%$ of its original concentration might be used successfully for monitoring PFF in survey, detection, monitoring and male annihilation technique (ElMetwally, 2014). On the other side, Sunflower oil $70 \%$ was used for diluting some of the hydrogenated samples for their physical characteristics(DeMan, et al., 1993).

The National Area Wide Eradication Program of $B$. zonata reqires usage of huge quantities of methyl eugenol in Egypt, therefore, there is a necessity to minimize the increasable costs of its applications. Reduction of the used quantity of methyl eugenol through diluting by paraffin oil or others seems to be one of the suggested solutions. Therefore, this work was conducted to evaluate the field efficiency of methyl eugenol and some oils mixtures for attraction of $B$. zonata males under Egyptian conditions.

\section{MATERIALS AND METHODS}

This study was designed in order to investigate the field efficiency of methyl eugenol mixed with certain oils for attracting the males of peach fruit fly through conducting two trails; firstly, testing the efficiency of different quantities of methyl eugenol and Sumithion mixture, secondly, investigation the efficiency of methyl eugenol diluted by corn, sunflower and paraffin oils. 


\section{A. Experimental locations and duration of trials:}

This study was carried out in 3 governorates which varied in their locations and climatic factors, Fayoum (at the north of Upper Egypt region), Dakhlia (at north Delta region) and Menofia (at middle Delta region).

- At Fayoum governorate, the selected orchard was located in Dekm village, Sinnorus District $\left(29.18{ }^{\circ} \mathrm{N}, 30.41^{\circ} \mathrm{E}\right)$ with a total area of 70 feddans. The selected location was cultivated with apricot, mango, limon, olive and mandarin. The $1^{\text {st }}$ trail started on July $2^{\text {nd }} 2014$, and continued for successive ten weeks, while the $2^{\text {nd }}$ trail started on September $5^{\text {th }}$ and continued for 10 weeks at the same year.

-At Dakhlia governorate, the experimental orchard was located at Mansoura district $\left(31.35{ }^{\circ} \mathrm{N}, 31.03^{\circ} \mathrm{E}\right)$, in area of about 9 feddans cultivated with guava (Psidium guajava). The $1^{\text {st }}$ trail started on September $10^{\text {th }}, 2014$ and continued for successive ten weeks.

-At Menofia governorate, the experimental orchard was located at Shibin Elkoam district $\left(31.0{ }^{\circ} \mathrm{N}, 30.55^{\circ} \mathrm{E}\right)$. The selected orchards were cultivated with guava (Psidium guajava) with area of about 14 feddans. The trail was started on September $9^{\text {th }}, 2014$ and continued for successive 9 weeks,

\section{B- Blocks treatment:}

a-The $1^{\text {st }}$ Trail:

To assess the maximum quantity of methyl eugenol that could be absorbed by the fiber blocks. The fiber blocks (measuring $5 \times 5 \times 1.1 \mathrm{~cm}$ ) were weighed before saturation with a mixture of methyl eugenol (Methyl Eugenol, 98\% purity ,Yasho Industries ${ }^{\circledR}$, India) and Sumithion as a toxicant at ratio of $4: 1$ for about four hours in the laboratory. After impregnation, the treated blocks were weighted again to record the quantity of absorbed soluation. Methyl eugenol quantities in weight were inverted to equivalent volume quantities depending on methyl eugenol density $(1.0356 \mathrm{gm} /$ $\mathrm{cm}^{3}$ ) (Lewis 2001). The mean of absorbed quantity was $8.00 \pm 0.25 \mathrm{~cm}^{3}$ for each block. The blocks were saturated with quantities of 6,4 and $2 \mathrm{~cm}^{3}$ to be compared with $8 \mathrm{~cm}^{3}$.

b-The $2^{\text {nd }}$ Trail:

The fiber blocks were treated with different concentrations of methyl eugenol $98,75,50$ and $25 \%$ mixed with technical Sumthion, and diluted using certain oils (paraffin, corn and sunflower. The impregnated blocks were transferred to the field on plastic bags. To collect the dead insects, plastic containers (measuring $20 \mathrm{~cm}$ in height and $10 \mathrm{~cm}$ in diameter) were fixed under the treated blocks by a metallic wire for receiving the dead male flies. The lured and killed $B$. zonata males in the plastic containers were weekly recorded without renewal of treatments. Seven replicates (7 
blocks / each treatment) were used and distributed in a complete randomized blocks design. All blocks were uniformly distributed and hanged at a height of $175 \mathrm{~cm}$ at least on the southern external branches of trees (in a shadow and aerated places on trees). The distance between every two adjacent blocks was about 65 meters to avoid interactions among blocks.

\section{C- Statistical analysis :}

Statistical analysis was conducted using one way ANOVA; (CosStat, 1990).

\section{RESULTS AND DISCUSSION}

I- $\quad$ Efficacy of different quantities of methyl eugenol on attraction of $B$. zonata males:

Data represented in Tables 1 and 2 shows the attracted mean numbers of peach fruit fly males attracted to blocks saturated with different quantities of methyl eugenol $\left(8,6,4\right.$ and $\left.2 \mathrm{~cm}^{3}\right)$ in two governorates (Fayoum and Dakhlia).

1- At Fayoum governorate :

As shown in Table 1, the captured flies of peach fruit fly male means of the treatments were significantly varied throughout of the evaluation period (10 weeks). Nonsignificant differences among the four tested methyl eugenol quantities in blocks were noticed within the weeks $1^{\text {st }}, 4^{\text {th }}$, and $8^{\text {th }}$, while, significant differences were observed during the rest of other weeks. The general mean no. of attracted flies throughout the 10 weeks of trail showed that there was nonsignificant difference between 8 and $6 \mathrm{~cm}^{3}$ treatments(13.46 and 18.55 flies / week, respectively) as well as between treatments 4 and $2 \mathrm{~cm}^{3}$ (8.46 and 4.99 flies / week, respectively), while there were significant differences between the two groups.

The respective means $\%$ of relative attractancy were $32.05,42.56,16.36$ and $9.03 \%$ throughout the studied period. The highest mean no. of captured flies was recorded during the $2^{\text {nd }}$ week by $8 \mathrm{~cm}^{3}$ methyl eugenol treated blocks (87.14 flies/week) with mean $\%$ of relative attractancy $47.92 \%$ coinciding with the highest recorded mean number of attracted males ( $31.60 \%$ of total captured flies), while, the lowest mean $\%$ of attracted flies $(2.41 \%)$ were observed during the $9^{\text {th }}$ week of the study.

2- At Dakhlia governorate :

As shown in Table 2, the mean no. of captured peach fruit fly males were significantly differed throughout of the evaluation period (10 weeks). Nonsignificant differences were observed among the four tested quantities of methyl eugenol within the first two weeks of the treatment. The general mean no. of attracted male flies of 
tested treatments $\left(8,6,4\right.$ and $\left.2 \mathrm{~cm}^{3}\right)$ throughout the 10 weeks were 55.67, 34.60, 25.20 and 13.51 flies / week with respective means \% of relative attractancy of 43.81 , $26.39,19.06$ and $10.74 \%$. The highest mean no of captured flies was recorded during the $7^{\text {th }}$ week by $8 \mathrm{~cm}^{3}$ methyl eugenol treated blocks ( 84.76 flies/week) with a mean relative attraction of $56.29 \%$. Throughout the studied period (10 weeks), the highest mean relative attraction $\%$ males was recorded in the $3^{\text {rd }}$ week $(13.31 \%)$, while, the lowest value was observed in the last week of the study $(6.03 \%)$.

Generally, results obtained in both governorates revealed the similar trend of response, statistical analysis indicated that the highest quantity of methyl eugenol(8 $\mathrm{cm}^{3)}$ ) was significantly higher than those of 6,4 , and $2 \mathrm{~cm}^{3}$ at Dakhlia governorate, while at Fayoum governorate, there was nonsignificant difference between 8 and 6 $\mathrm{cm}^{3}$ treatments.

Table 1. Mean no. of peach fruit fly males attracted to different quantities of methyl eugenol in fiber blocks at Fayoum governorate during season 2014.

\begin{tabular}{|c|c|c|c|c|c|c|c|c|c|c|}
\hline \multirow[b]{2}{*}{ Weeks } & \multicolumn{5}{|c|}{ Means no. of attracted flies (flies / week) } & \multicolumn{4}{|c|}{ Relative attraction $\%$} & \multirow{2}{*}{$\begin{array}{c}\text { Weekly } \\
\% \text { of } \\
\text { total } \\
\text { attracted } \\
\text { flies }\end{array}$} \\
\hline & $\begin{array}{l}8 \mathrm{~cm}^{3} / \\
\text { Block }\end{array}$ & $\begin{array}{l}6 \mathrm{~cm}^{3} / \\
\text { Block }\end{array}$ & $\begin{array}{l}4 \mathrm{~cm}^{3} / \\
\text { Block }\end{array}$ & $\begin{array}{l}2 \mathrm{~cm}^{3} / \\
\text { Block }\end{array}$ & $\mathrm{LSD}_{05}$ & $\begin{array}{c}8 \\
\mathrm{~cm}^{3} / \\
\text { Block }\end{array}$ & $\begin{array}{c}6 \\
\mathrm{~cm}^{3} / \\
\text { Block }\end{array}$ & $\begin{array}{c}4 \\
\mathrm{~cm}^{3} / \\
\text { Block }\end{array}$ & $\begin{array}{c}2 \\
\mathrm{~cm}^{3} / \\
\text { Block }\end{array}$ & \\
\hline $1^{\text {st }}$ & $29.00 \mathrm{a}$ & $21.00 \mathrm{a}$ & $13.14 \mathrm{a}$ & $16.67 \mathrm{a}$ & NS & 36.34 & 26.31 & 16.46 & 20.89 & 17.56 \\
\hline $2^{\text {nd }}$ & $28.20 \mathrm{ab}$ & $36.60 \mathrm{a}$ & $15.40 \mathrm{bc}$ & $8.80 \mathrm{c}$ & 13.24 & 31.69 & 41.12 & 17.30 & 9.89 & 19.58 \\
\hline $3^{\text {rd }}$ & $16.80 \mathrm{ab}$ & $25.00 \mathrm{a}$ & $18.60 \mathrm{ab}$ & $5.80 \mathrm{c}$ & 13.86 & 25.38 & 37.76 & 28.10 & 8.76 & 14.57 \\
\hline $4^{\text {th }}$ & $11.43 \mathrm{a}$ & $13.71 \mathrm{a}$ & 7.57 a & $6.86 \mathrm{a}$ & NS & 28.89 & 34.65 & 19.13 & 17.34 & 8.71 \\
\hline $5^{\text {th }}$ & $10.57 b$ & $25.86 \mathrm{a}$ & $5.86 \mathrm{~b}$ & $2.86 \mathrm{~b}$ & 14.21 & 23.41 & 57.28 & 12.98 & 6.33 & 9.94 \\
\hline $6^{\text {th }}$ & $10.43 a b$ & $17.71 \mathrm{a}$ & $3.71 \mathrm{~b}$ & $1.29 \mathrm{~b}$ & 11.92 & 31.47 & 53.44 & 11.19 & 3.89 & 7.29 \\
\hline $7^{\text {th }}$ & $8.43 \mathrm{a}$ & $13.86 \mathrm{a}$ & $9.00 \mathrm{a}$ & $2.43 \mathrm{~b}$ & 11.42 & 25.00 & 41.10 & 26.69 & 7.21 & 7.42 \\
\hline $8^{\text {th }}$ & $12.86 \mathrm{a}$ & $13.00 \mathrm{a}$ & $5.14 \mathrm{a}$ & 3.29 a & NS & 37.50 & 37.91 & 14.99 & 9.59 & 7.55 \\
\hline $9^{\text {th }}$ & $2.71 b$ & 9.29 a & $1.57 \mathrm{~b}$ & $0.29 \mathrm{~b}$ & 5.34 & 19.55 & 67.03 & 11.33 & 2.09 & 3.05 \\
\hline $10^{\text {th }}$ & $4.14 a b$ & $9.43 \mathrm{a}$ & $4.57 a b$ & $1.57 \mathrm{~b}$ & 5.80 & 21.00 & 47.84 & 23.19 & 7.97 & 4.34 \\
\hline Mean & $13.46 \mathrm{AB}$ & $18.55 \mathrm{~A}$ & $8.46 \mathrm{~B}$ & $4.99 \mathrm{BC}$ & 6.56 & 32.05 & 42.56 & 16.36 & 9.03 & \\
\hline
\end{tabular}

Means followed by the same letter are not significantly different at the .05 level for each row. 
Table 2. Mean no. of peach fruit fly males attracted to different quantities of methyl eugenol in fiber blocks at Dakhlia governorate during season 2014.

\begin{tabular}{|c|c|c|c|c|c|c|c|c|c|c|}
\hline \multirow{2}{*}{ Weeks } & \multicolumn{5}{|c|}{ Means no. of attracted flies (flies /week) } & \multicolumn{4}{|c|}{ Relative attraction \% } & \multirow{2}{*}{$\begin{array}{l}\text { Weekly \% } \\
\text { of total } \\
\text { attracted } \\
\text { flies }\end{array}$} \\
\hline & $\begin{array}{c}8 \mathrm{~cm}^{3} / \\
\text { Block }\end{array}$ & $\begin{array}{c}6 \mathrm{~cm}^{3} / \\
\text { Block }\end{array}$ & $\begin{array}{l}4 \mathrm{~cm}^{3} / \\
\text { Block }\end{array}$ & $\begin{array}{l}2 \mathrm{~cm}^{3} / \\
\text { Block }\end{array}$ & $\operatorname{LSD}_{05}$ & $\begin{array}{l}8 \mathrm{~cm}^{3} / \\
\text { Block }\end{array}$ & $\begin{array}{c}6 \\
\mathrm{~cm}^{3} / \\
\text { Block }\end{array}$ & $\begin{array}{c}4 \\
\mathrm{~cm}^{3} / \\
\text { Block }\end{array}$ & $\begin{array}{l}2 \mathrm{~cm}^{3} / \\
\text { Block }\end{array}$ & \\
\hline $1^{\mathrm{st}}$ & $22.96 \mathrm{a}$ & $23.60 \mathrm{a}$ & 22.96 a & $24.71 \mathrm{a}$ & NS & 24.37 & 25.05 & 24.37 & 26.22 & 7.31 \\
\hline $2^{\text {nd }}$ & $31.50 \mathrm{a}$ & $49.03 \mathrm{a}$ & 41.75 a & $24.07 a$ & NS & 21.52 & 33.50 & 28.53 & 16.45 & 11.35 \\
\hline $3^{\text {rd }}$ & $72.82 \mathrm{a}$ & $44.39 \mathrm{~b}$ & $35.71 b$ & $18.75 \mathrm{c}$ & 16.09 & 42.42 & 25.86 & 20.80 & 10.92 & 13.31 \\
\hline $4^{\text {th }}$ & $64.53 \mathrm{a}$ & $46.28 \mathrm{~b}$ & $33.82 \mathrm{bc}$ & $21.74 \mathrm{c}$ & 14.79 & 38.79 & 27.82 & 20.33 & 13.07 & 12.90 \\
\hline $5^{\text {th }}$ & $65.82 \mathrm{a}$ & $31.32 \mathrm{~b}$ & $32.78 \mathrm{~b}$ & $9.53 c$ & 18.22 & 47.20 & 22.46 & 23.51 & 6.83 & 10.81 \\
\hline $6^{\text {th }}$ & $46.46 a$ & $53.35 \mathrm{a}$ & $17.28 \mathrm{~b}$ & $11.46 \mathrm{~b}$ & 18.92 & 36.14 & 41.50 & 13.44 & 8.91 & 9.97 \\
\hline $7^{\text {th }}$ & $84.46 \mathrm{a}$ & $33.78 \mathrm{~b}$ & $25.03 \mathrm{bc}$ & $6.78 \mathrm{c}$ & 18.92 & 56.29 & 22.51 & 16.68 & 4.52 & 11.63 \\
\hline $8^{\text {th }}$ & $51.78 \mathrm{a}$ & $21.17 \mathrm{~b}$ & $12.14 \mathrm{~b}$ & $8.82 \mathrm{~b}$ & 20.93 & 55.14 & 22.54 & 12.93 & 9.39 & 7.28 \\
\hline $9^{\text {th }}$ & $72.28 \mathrm{a}$ & $27.60 \mathrm{~b}$ & 19.78 bc & $1.78 \mathrm{c}$ & 21.42 & 59.52 & 22.73 & 16.29 & 1.47 & 9.42 \\
\hline $10^{\text {th }}$ & $44.10 \mathrm{a}$ & $15.53 \mathrm{~b}$ & $10.71 b$ & $7.46 b$ & 15.16 & 56.68 & 19.96 & 13.77 & 9.59 & 6.03 \\
\hline Mean & $55.67 \mathrm{~A}$ & $34.61 \mathrm{~B}$ & $25.20 \mathrm{BC}$ & $13.51 \mathrm{C}$ & 12.26 & 43.81 & 26.39 & 19.06 & 10.74 & \\
\hline
\end{tabular}

Means followed by the same letter are not significantly different at the .05 level for each row.

II - Efficacy of methyl eugenol diluted with certain oils on attraction of peach fruit fly males:

Dilutions of methyl eugenol with different oils including corn, sunflower and paraffin oils at two different ratios (75 and 50\%) were evaluated in two different governorates (Fayoum and Menofia) by applying male annihilation technique. The evaluated treatments included; (A) Methyl eugenol 98\% , (B) Methyl eugenol $75 \%+$ corn oil 25\%, (C) Methyl eugenol 50\% + corn oil 50\%, (D) Methyl eugenol $75 \%+$ sunflower oil 25\%, (E) Methyl eugenol $50 \%+$ sunflower oil 50\%, (F) Methyl eugenol $75 \%$ + paraffin oil 25\%, and (G) Methyl eugenol $50 \%$ + paraffin oil $50 \%$.

-At Fayoum governotare :

As shown in Table (3): the weekly mean no. of captured males varied significantly during the $1^{\text {st }}, 2^{\text {nd }}, 4^{\text {th }}, 5^{\text {th }}, 6^{\text {th }}$ and $7^{\text {th }}$ weeks of inspection, while, nonsignificant differences were observed during the rest weeks. The highest mean no. 
of captured flies was recorded by the $\mathrm{G}$ treatment ( $50 \%$ paraffin $+50 \%$ methyl eugenol) on the $2^{\text {nd }}$ week.

Among the tested treatments, the general mean no. of captured flies/week throughout ten weeks in treatment, $G$ (35.85), $F(24.86)$ and $B(24.78)$ were significantly higher than the standard treatment A (20.65). Such the obtained results indicated regarding the average of relative attractancy \% (Table 4), that the treatment $G(23.76 \%)$, the treatment $F(19.94 \%)$ and the treatment $B(16.89)$ showed the highest capturing comparing to the standard treatment A (14.07\%).

- At Menofia governorate:

As shown in table (5), the weekly mean no. of captured males varied insignificantly during all the nine weeks of investigation except at $4^{\text {th }}$ and $5^{\text {th }}$ weeks. The highest mean no. of captured flies/week (43.40)

Table 3. Mean no. of $B$. zonata males attracted to blocks treated with different dilutions of methyl eugenol at Fayoum governorate during season 2014.

\begin{tabular}{|c|c|c|c|c|c|c|c|c|}
\hline \multirow{3}{*}{ Weeks } & \multicolumn{8}{|c|}{ Means of attracted flies (flies / week) } \\
\hline & \multirow{2}{*}{$\begin{array}{c}\text { A } \\
\text { (standard } \\
\text { treatment) }\end{array}$} & \multicolumn{2}{|c|}{ Corn oil } & \multicolumn{2}{|c|}{ Sunflower oil } & \multicolumn{2}{|c|}{ Paraffin oil } & \multirow{2}{*}{$\mathrm{LSD}_{05}$} \\
\hline & & $\mathrm{B}(75 \%)$ & $C(50 \%)$ & $\mathrm{D}(75 \%)$ & $E(50 \%)$ & $F(75 \%)$ & $\mathrm{G}(50 \%)$ & \\
\hline $1^{\text {st }}$ & $33.00 a b$ & $54.83 a$ & $21.66 b$ & $25.00 \mathrm{~b}$ & $15.33 b$ & $39.16 \mathrm{ab}$ & $55.16 \mathrm{a}$ & 25.88 \\
\hline $2^{\text {nd }}$ & $64.00 \mathrm{ab}$ & 39.83 bc & $20.33 b c$ & 42.16 bc & $16.00 \mathrm{c}$ & $59.16 a b c$ & $94.50 \mathrm{a}$ & 40.65 \\
\hline $3^{\text {rd }}$ & $17.83 \mathrm{a}$ & $18.33 \mathrm{a}$ & $19.50 \mathrm{a}$ & $26.00 \mathrm{a}$ & $15.16 \mathrm{a}$ & $18.00 \mathrm{a}$ & $35.50 \mathrm{a}$ & 27.93 \\
\hline $4^{\text {th }}$ & $29.33 a b$ & $31.33 a b$ & $12.83 \mathrm{c}$ & $24.00 \mathrm{abc}$ & $18.66 \mathrm{bc}$ & $31.83 a b$ & $39.16 a$ & 17.71 \\
\hline $5^{\text {th }}$ & $21.66 \mathrm{~b}$ & $31.33 \mathrm{ab}$ & $15.50 \mathrm{~b}$ & $26.33 \mathrm{ab}$ & $15.00 \mathrm{~b}$ & $28.66 \mathrm{ab}$ & $44.66 \mathrm{a}$ & 14.69 \\
\hline $6^{\text {th }}$ & $12.16 \mathrm{~b}$ & $17.50 \mathrm{~b}$ & $5.50 \mathrm{~b}$ & $9.50 \mathrm{~b}$ & $13.00 \mathrm{~b}$ & $16.60 \mathrm{~b}$ & $30.66 \mathrm{a}$ & 11.54 \\
\hline $7^{\text {th }}$ & $3.66 \mathrm{ab}$ & $9.83 a b$ & $7.83 a b$ & $3.00 a b$ & $0.83 \mathrm{~b}$ & $17.00 \mathrm{a}$ & $16.83 \mathrm{a}$ & 9.66 \\
\hline $8^{\text {th }}$ & $11.66 \mathrm{a}$ & $16.66 \mathrm{a}$ & $13.83 \mathrm{a}$ & $12.00 \mathrm{a}$ & $2.83 \mathrm{a}$ & $13.00 \mathrm{a}$ & $17.00 \mathrm{a}$ & NS \\
\hline $9^{\text {th }}$ & $9.66 \mathrm{a}$ & $10.66 \mathrm{a}$ & $4.50 \mathrm{a}$ & $4.16 \mathrm{a}$ & $3.33 \mathrm{a}$ & $8.00 \mathrm{a}$ & $3.00 \mathrm{a}$ & NS \\
\hline $10^{\text {th }}$ & $8.50 \mathrm{a}$ & $17.50 \mathrm{a}$ & $8.33 \mathrm{a}$ & $8.66 \mathrm{a}$ & $4.83 a$ & 17.66 a & $9.33 \mathrm{a}$ & NS \\
\hline Mean & $20.65 \mathrm{AB}$ & $24.78 \mathrm{AB}$ & $12.98 \mathrm{~B}$ & $18.08 \mathrm{AB}$ & $10.50 \mathrm{~B}$ & $24.86 \mathrm{AB}$ & $34.85 \mathrm{~A}$ & 12.19 \\
\hline
\end{tabular}

Means followed by the same letter are not significantly different at the .05 level for each row. 
Table 4. Relative attractancy of $B$. zonata males attracted to blocks treated with different dilutions of methyl eugenol at Fayoum governorate during season 2014.

\begin{tabular}{|c|c|c|c|c|c|c|c|c|}
\hline \multirow{3}{*}{ Weeks } & \multicolumn{7}{|c|}{ Relative attractancy (\%) } & \multirow{3}{*}{$\begin{array}{c}\text { Weekly \% } \\
\text { of total } \\
\text { attracted } \\
\text { flies }\end{array}$} \\
\hline & \multirow{2}{*}{$\begin{array}{c}\text { A } \\
\text { (standar } \\
\text { d } \\
\text { treatme } \\
\text { nt) }\end{array}$} & \multicolumn{2}{|c|}{ Corn oil } & \multicolumn{2}{|c|}{ Sunflower oil } & \multicolumn{2}{|c|}{ Paraffin oil } & \\
\hline & & $\mathrm{B}(75 \%)$ & $C(50 \%)$ & $\mathrm{D}(75 \%)$ & $E(50 \%)$ & $F(75 \%)$ & $\begin{array}{c}G(50 \% \\
\quad)\end{array}$ & \\
\hline $1^{\text {st }}$ & 13.52 & 22.46 & 8.87 & 10.24 & 6.28 & 16.04 & 22.59 & 16.61 \\
\hline $2^{\text {nd }}$ & 19.05 & 11.85 & 6.05 & 12.56 & 4.75 & 17.61 & 28.13 & 22.86 \\
\hline $3^{\text {rd }}$ & 11.86 & 12.19 & 12.97 & 17.3 & 10.09 & 11.97 & 23.62 & 10.23 \\
\hline $4^{\text {th }}$ & 15.67 & 16.74 & 6.86 & 12.82 & 9.97 & 17.01 & 20.93 & 12.73 \\
\hline $5^{\text {th }}$ & 11.83 & 17.11 & 8.46 & 14.37 & 8.19 & 15.65 & 24.39 & 12.46 \\
\hline $6^{\text {th }}$ & 11.59 & 16.68 & 5.24 & 9.06 & 12.39 & 15.82 & 29.22 & 7.14 \\
\hline $7^{\text {th }}$ & 6.20 & 16.67 & 13.28 & 5.09 & 1.41 & 28.82 & 28.53 & 4.01 \\
\hline $8^{\text {th }}$ & 13.41 & 19.15 & 15.9 & 13.80 & 3.25 & 14.95 & 19.54 & 5.92 \\
\hline $9^{\text {th }}$ & 22.30 & 24.61 & 10.39 & 9.61 & 7.69 & 18.47 & 6.93 & 2.947 \\
\hline $10^{\text {th }}$ & 11.36 & 23.39 & 11.13 & 11.58 & 6.46 & 23.61 & 12.47 & 5.09 \\
\hline Mean & 14.07 & 16.89 & 8.85 & 12.32 & 7.16 & 19.94 & 23.76 & \\
\hline
\end{tabular}

Table 5. Mean no. of $B$. zonata males attracted to blocks treated with different dilutions of methyl eugenol at Menofia governorate during season 2014.

\begin{tabular}{|c|c|c|c|c|c|c|c|c|}
\hline \multirow{3}{*}{ Weeks } & \multicolumn{8}{|c|}{ Means of attracted flies (flies /week) } \\
\hline & \multirow{2}{*}{$\begin{array}{c}\text { A } \\
\text { (standard } \\
\text { treatment) }\end{array}$} & \multicolumn{2}{|c|}{ Corn oil } & \multicolumn{2}{|c|}{ Sunflower oil } & \multicolumn{2}{|c|}{ Paraffin oil } & \multirow[b]{2}{*}{$\mathrm{LSD}_{05}$} \\
\hline & & $B(75 \%)$ & $C(50 \%)$ & $\mathrm{D}(75 \%)$ & $B(75 \%)$ & $C(50 \%)$ & $\mathrm{D}(75 \%)$ & \\
\hline $1^{\text {st }}$ & $6.00 \mathrm{a}$ & $7.20 \mathrm{a}$ & $5.40 \mathrm{a}$ & $5.80 \mathrm{a}$ & $5.20 \mathrm{a}$ & $8.80 \mathrm{a}$ & $9.00 \mathrm{a}$ & NS \\
\hline $2^{\text {nd }}$ & $8.40 \mathrm{a}$ & $6.80 \mathrm{a}$ & $5.00 \mathrm{a}$ & $6.80 \mathrm{a}$ & $6.20 \mathrm{a}$ & $5.60 \mathrm{a}$ & $5.80 \mathrm{a}$ & NS \\
\hline $3^{\text {rd }}$ & $10.40 \mathrm{a}$ & $8.20 \mathrm{a}$ & $8.20 \mathrm{a}$ & $7.80 \mathrm{a}$ & $9.40 \mathrm{a}$ & $8.60 \mathrm{a}$ & $8.20 \mathrm{a}$ & NS \\
\hline $4^{\text {th }}$ & $12.20 \mathrm{a}$ & $8.00 a b$ & $7.60 a b$ & $5.80 \mathrm{~b}$ & $\begin{array}{c}11.00 \\
a b\end{array}$ & $6.00 \mathrm{~b}$ & $9.20 a b$ & 5.03 \\
\hline $5^{\text {th }}$ & $17.40 \mathrm{a}$ & $\begin{array}{c}10.80 \\
a b\end{array}$ & $8.80 a b$ & $5.60 \mathrm{~b}$ & $\begin{array}{c}10.20 \\
a b\end{array}$ & $7.20 \mathrm{ab}$ & $\begin{array}{c}13.20 \\
a b\end{array}$ & 9.67 \\
\hline $6^{\text {th }}$ & $16.00 \mathrm{a}$ & $21.80 \mathrm{a}$ & $9.20 \mathrm{a}$ & $10.20 \mathrm{a}$ & $19.20 \mathrm{a}$ & $10.60 \mathrm{a}$ & $16.20 \mathrm{a}$ & NS \\
\hline $7^{\text {th }}$ & $43.40 \mathrm{a}$ & $26.60 \mathrm{a}$ & $31.00 \mathrm{a}$ & $29.20 \mathrm{a}$ & $35.40 \mathrm{a}$ & $22.00 \mathrm{a}$ & $23.00 \mathrm{a}$ & NS \\
\hline $8^{\text {th }}$ & $14.00 \mathrm{a}$ & $13.00 \mathrm{a}$ & $8.40 \mathrm{a}$ & $9.80 \mathrm{a}$ & $15.20 \mathrm{a}$ & $5.00 \mathrm{a}$ & $14.00 \mathrm{a}$ & NS \\
\hline $9^{\text {th }}$ & $21.50 \mathrm{a}$ & $12.00 \mathrm{a}$ & $5.50 \mathrm{a}$ & $10.00 \mathrm{a}$ & $8.75 \mathrm{a}$ & $6.75 \mathrm{a}$ & $17.25 \mathrm{a}$ & NS \\
\hline Mean & $17.83 \mathrm{~A}$ & $11.19 \mathrm{~A}$ & $12.55 \mathrm{~A}$ & $9.91 \mathrm{~A}$ & $9.74 \mathrm{~A}$ & $11.50 \mathrm{~A}$ & $16.58 \mathrm{~A}$ & NS \\
\hline
\end{tabular}

Means followed by the same letter are not significantly different at the .05 level for each row. 
Table 6. Relative attractancy of $B$. zonata males to different dilutions of methyl eugenol treated-blocks at Menofia governorate in season 2014.

\begin{tabular}{|c|c|c|c|c|c|c|c|c|}
\hline \multirow{3}{*}{ Weeks } & \multicolumn{7}{|c|}{ Relative attractancy $\%$} & \multirow{3}{*}{$\begin{array}{l}\text { Weekly \% of } \\
\text { total } \\
\text { attracted } \\
\text { flies }\end{array}$} \\
\hline & \multirow{2}{*}{$\begin{array}{c}\text { A } \\
\text { (standard } \\
\text { treatment) }\end{array}$} & \multicolumn{2}{|c|}{ Corn oil } & \multicolumn{2}{|c|}{ Sunflower oil } & \multicolumn{2}{|c|}{ Paraffin oil } & \\
\hline & & $\mathrm{B}(75 \%)$ & $C(50 \%)$ & $D(75 \%)$ & $B(75 \%)$ & $C(50 \%)$ & $D(75 \%)$ & \\
\hline $1^{\mathrm{st}}$ & 12.66 & 15.19 & 11.39 & 12.24 & 10.97 & 18.56 & 18.99 & 6.23 \\
\hline $2^{\text {nd }}$ & 18.83 & 15.25 & 11.21 & 15.25 & 13.90 & 12.56 & 13.00 & 5.86 \\
\hline $3^{\text {rd }}$ & 17.10 & 13.49 & 13.49 & 12.83 & 15.46 & 14.14 & 13.49 & 7.99 \\
\hline $4^{\text {th }}$ & 20.40 & 13.38 & 12.71 & 9.70 & 18.39 & 10.03 & 15.39 & 7.86 \\
\hline $5^{\text {th }}$ & 23.77 & 14.75 & 12.02 & 7.65 & 13.94 & 9.84 & 18.03 & 9.62 \\
\hline $6^{\text {th }}$ & 15.50 & 21.13 & 8.92 & 9.88 & 18.60 & 10.27 & 15.70 & 13.57 \\
\hline $7^{\text {th }}$ & 20.61 & 12.63 & 14.72 & 13.87 & 16.81 & 10.44 & 10.92 & 27.68 \\
\hline $8^{\text {th }}$ & 17.63 & 16.37 & 10.58 & 12.34 & 19.15 & 6.30 & 17.63 & 10.44 \\
\hline $9^{\text {th }}$ & 26.30 & 14.68 & 6.73 & 12.23 & 10.70 & 8.26 & 21.10 & 10.75 \\
\hline Mean & 19.96 & 12.53 & 14.05 & 11.10 & 10.91 & 12.88 & 18.57 & \\
\hline
\end{tabular}

was recorded by the A treatment ( $98 \%$ methyl eugenol) on the $7^{\text {th }}$ week. The general means throughout the studied period ( 9 weeks) varied insignificantly; the treatments $A$, treatment $G$ were and the treatment $C$ were relatively attracted higher numbers of flies in comparison with other treatments (17.83, 16.58 and 12.55 flies/ week, respectively) with mean relative attractancy \% of $19.96,18.57$ and $14.05 \%$.The highest weekly mean $(26.30 \%)$ was recorded by the treatment A (on the $9^{\text {th }}$ week).

The fiber blocks treated with methyl eugenol and Sumithion as a toxicant were the highest efficient for attracting and killing the highest numbers of $B$. zonata males for up to 4 weeks (Ghanim et. al., 2010 and Amin, 2013). Response of the tephritid fly, B. zonata to methyl eugenol-insecticide obviously varied according to methyl eugenol concentration. The blocks that impregnated with $8 \mathrm{~cm}^{3}$ (the maximium and standard quantitiy of methyl eugenol) captured significantly the highest number of $B$. zonata males in comparison with other treatments. The obtained results agree with that mentioned by Vargas et al., 2000 who evaluated the same quantities of methyl eugenol $\left(8,6,4\right.$ and $\left.2 \mathrm{~cm}^{3}\right)$ for capturing $B$. dorsails by using enclosed cotton wicks fixed internally plastic bucket traps. Also, Ravikumar 2006 found positive responses of the tephritid fly. B. zonata to methyl eugenol-insecticide as the quantity increased. However, among $0.8,0.6,0.4$ and $0.2 \mathrm{~cm}^{3}$ of methyl eugenol, traps charged with $0.4 \mathrm{ml}$ methyl eugenol was superior in attracting highest 
number of $B$. dorsalis and $B$. correcta. While, the highest number of $B$. zonata was recorded in the traps charged with $0.6 \mathrm{ml}$ methyl eugenol. When the total fruit flies irrespective of species were considered, traps charged with $0.4 \mathrm{ml}$ methyl eugenol significantly attracted more fruit flies. The lowest number of trap catches was recorded in the traps charged with $1.0 \mathrm{ml}$ methyl eugenol (Nagaraj et. al.,2014 ).

Because of its high economic costs involved with eradication of $B$. zonata in Egypt, dilution of methyl eugenol with paraffin oil of $50-75 \%$ appears to be promising that could be depended upon until ratio to save methyl eugenol used quantities. This finding is in agreement with previously reported by Ghanim 2013 who mentioned that dilution of methyl eugenol with paraffin oil till $50 \%$ in MAT by using spinosad (as an insecticide) did not significantly affect the captured males and had a relatively high effect against B. zonata population. Also, EL-Metwally (2014) indicated that dilutions of methyl eugenol with paraffin oil may cause slow and regular release of the volatile in its mixture and hence maintains potency for a longer time.

Depending upon previous results, it could be concluded that dilution of methyl eugenol with paraffin oil till $50-75 \%$ in MAT did not significantly affect the captured males of B. zonata population in comparison with corn and sunflower oils. Such procedure applications could save $20-35 \%$ of the total economic costs for detection, monitoring and controlling the peach fruit fly population. Supporting this target requires particularly further investigations on the effect of dilution of methyl eugenol in different applications on large scale is required, also, the effect of time of application, weather factors and host should be considered.

\section{REFERENCES}

1. Afzal, M. and Javed, H. 2001. Evaluation of soaked wooden killer blocks for Male Annihilation (MA) on fruit fly, Bactrocera spp. (Diptera: Tephritidae). J. Biol. Sci., 1: 577-579.

2. Afzal, M.; Mahmood, R. and Stonehouse, J. M. 2001. Soaked-wood killer blocks for MAT fruit fly control in Pakistan. Proceedings of the Indian Ocean Commission, Regional Fruit Fly Symposium, Flic en Flac, Mauritius, 5-9 June, 2000, pp. 97-100.

3. Amin, A. A. 2013. Efficiency of wooden blocks and cotton wicks as dispensers of methyl eugenol for attracting and kill the peach fruit fly males. J. Plant Prot. and Pathol., Mansoura Univ., Vol. 4 (3): 245 - 252.

4. CoStat Software .1990. Microcomputer program analysis Version 4.2, CoHort Sofware, Berkeley, CA. 
5. DeMan, L., Xu, Y. J., Chen, H. S. and DeMan, J. M. 1993. Polymorphic stability of hydrogenated palm oleins in dilutions with unhydrogenated liquid oils. Journal of the American Oil Chem. Soc.70(4), 431-433.

6. El-Metwally, M. M. 2014. Response of the peach fruit fly, Bactrocera zonata (Saunders) (Diptera: Tephritidae) males to different concentrations of methyl eugenol under field conditions in Egypt.Bull. Ent. Soc. Egypt (in press).

7. Eisemann, C. H. and Rice, M. J. .1992. Attractants for the gravid Queensland fruit fly, Dacu stryoni. Entomol. Exp. Appl., 62: 125-130.

8. Ghanim, N. M. 2013. Influence of methyl eugenol diluted with paraffin oil on male annihilation technique of peach fruit fly, Bactrocera zonata (Saunders) (Diptera: Tephritidae). Entomol. Ornithol. Herpetol., 2: 114.

9. Ghanim, N. M., Moustafa, S. A., El-Metwally, M. M., Afia, Y.E. and Salman, M.S. 2010. Efficiency of some insecticides in male annihilation technique of peach fruit fly, Bactrocera zonata (Saunders) under Egyptian conditions. Egypt. Acad. J. Biolog. Sci. 2: 13-19.

10. Lewis, R. J. Sr. 2001. Hawley's condensed chemical dictionary. $14^{\text {th }}$ ed. New York (NY), John Wiley \& Sons, pp 735.

11. Metcalf, R. L. and Metcalf, E. R. 1992. Plant Kairomones in Insect Control. Chapman and Hall, New York, NY, USA.

12. Nagaraj, K.S., Jaganath, S., Srikanth, L. and Mahamadtoufeeq, H. 2014. Attraction of fruit Fly to different quantities of methyl eugenol in mango orchard. Trends in Biosciences, 7(11);1113-1115.

13. Vargas, R. I., Stark, J. D., Kido, M. H. Ketter, H. M. and Whiteh, L.C. 2000. Methyl eugenol and cue-lure traps for suppression of male oriental fruit flies and melon flies (Diptera: Tephritidae) in Hawaii: effects of lure mixtures and weathering. J. Econ. Entomol, 93: 81-87.

14. Qureshi, Z.A., Bughio, A.R. and Siddiqui, Q. H. 1981. Population suppression of fruit fly, Dacus zonatus (Saund) (Dipt., Teph.) by male annihilation technique and its impact on fruit infestation. Z. ang. Ent., 91: 521-524.

15. Ravikumar, P. 2006. Studies on fruit fly trapping systems by using methyl eugenol and protein food baits in guava and mango orchards. (M. Sc. Thesis, fac. Agric. Sci., Dharwad Univ., India).

16. Zaheeruddin, M. 2007. Study of diffusion and adoption of Male Annihilation Technique. International J. Education and Development using Information and Communication Technol. (IJEDICT), 3 (2): 89-99. 


\section{تأثير تخفيف الميثيل ايوجينول ببعض الزيوت على جذب ذبابة ثمار الخوخ في الحقل} مصطفى مهران المتولي و علي أحمد أمين

$$
\text { معرة بحوث وقاية النباتات - مركز البحوث الزراعية - الدقي - الجيزة - مصر }
$$

تعتبر ذبابة ثمار الخوخ من الحشرات الهامة إقتصاديا على ثمار الفاكهة في مصر ، وتعتبر

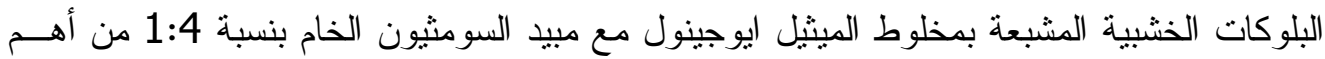

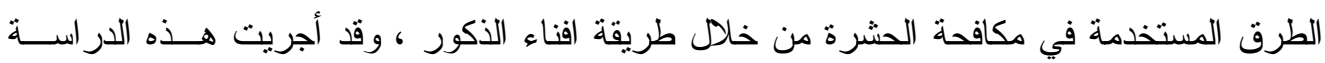

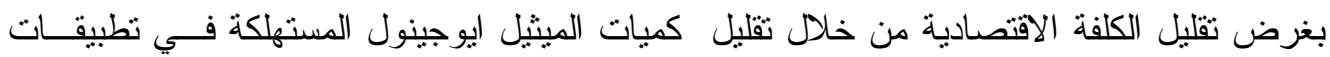

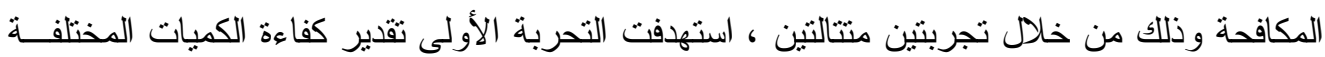

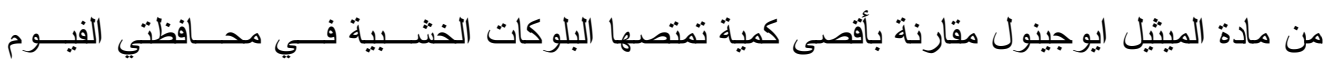
و الدقهلية. وقد تم مقارنة الاعداد المنجذبة الى البلوكات المشبعة بكمية 8 سم ${ }^{3}$ (أقصى كمية تمنصهية

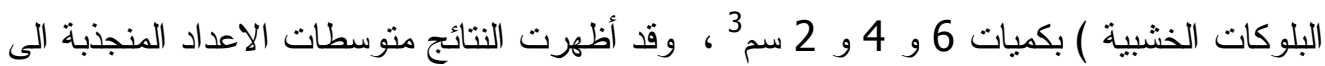

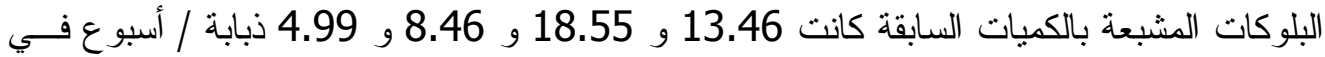

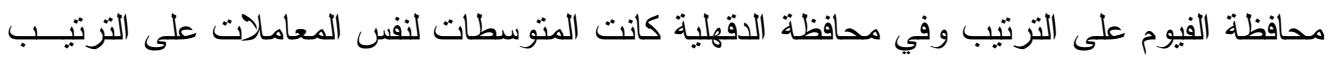

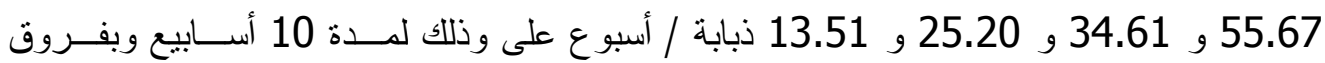

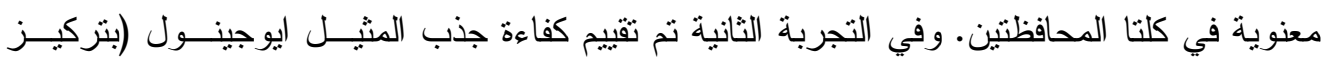

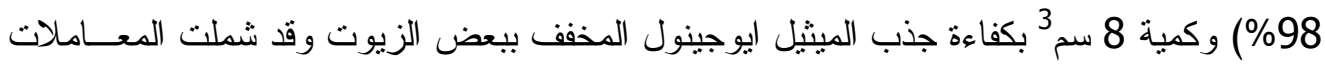
المختبرة الآتي : بلوكات معاملة بمخلوط المينيل ايوجينول وبلوكات معاملة بمادة المينيل ايوجينول

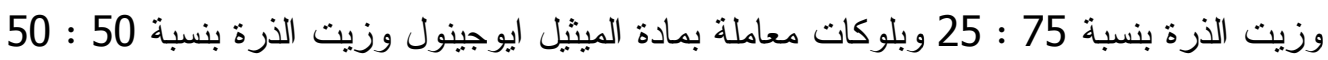
و وبلوكات معاملة بمادة الميثيل ايوجينول وزيت عباد الثمس بنسبة 75 : 25 وبلوكات معاملـــة

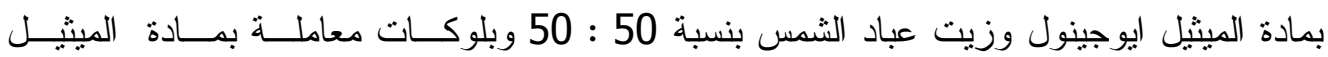
ايوجينول وزيت البر افين بنسبة 75 : 25 وبلوكات معاملة بمادة الميثيل ايوجينول وزيت وليت البـــر افين بنسبة 50 : 50. وقد أظهرت النتائج وجود اختلافات معنوية بين متوسطات الاعداد المنجذبة الــى

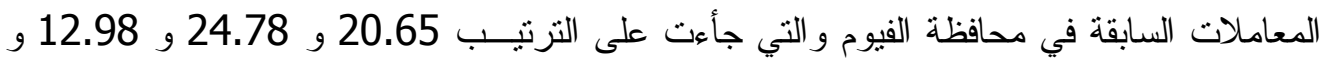

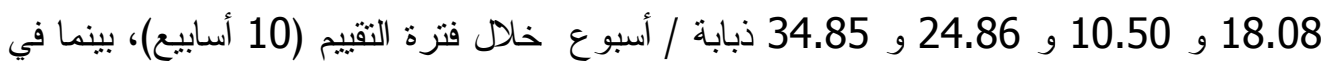

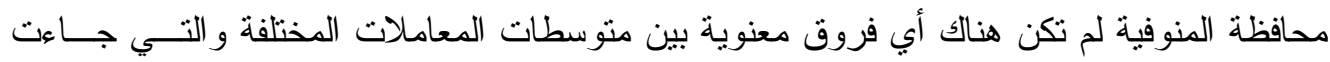

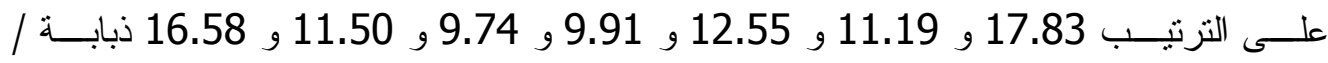
أسبوع خلال فترة التقييم (9 أسابيع)، ، و أظهرت النتائج في كلتا المحافظتين أنه يمكن الاعتماد على ولى ولى ولئ

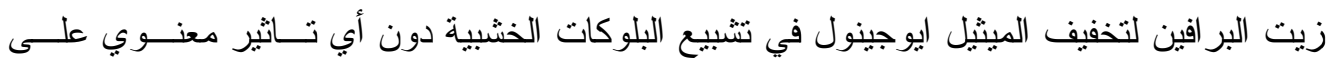

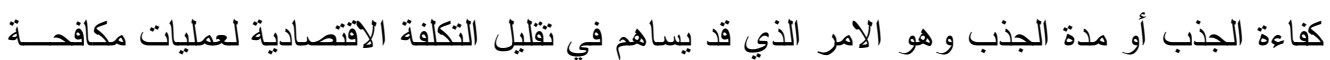

